\title{
Controversy over exercise therapy for chronic fatigue syndrome: key lessons for clinicians and academics
}

ROUND THE CORNER

\author{
Alex J. Mitchell \\ COMMENTARY ON... COCHRANE CORNER ${ }^{\dagger}$
}

\begin{abstract}
SUMMARY
Chronic fatigue syndrome (CFS) is syndrome of unremitting fatigue of at least 6 months' duration that causes signific ant disability. Exercise therapy has a proven track record in medicine and could be effective for some patients with CFS. An updated Cochrane review of eight studies appeared to suggest that exercise helps fatigue symptoms, but with only a small probability of recovery and/ or improvement in daily function. Provisional data on acceptability suggest that most patients are willing to participate. However, one key study (PACE), which was well powered and influential in the Cochrane review, has been met with considerable controversy owing to lack of clarity on outcomes. Following release of the PACE study primary data, re-analysis suggested smaller effect sizes than initially reported.
\end{abstract}

\section{DECLARATION OF INTEREST}

None

Chronic fatigue syndrome (CFS) is a recognised but poorly understood condition for which there is no single recognised cause. The hallmark is persistent unremitting fatigue lasting at least 6 months and causing significant disability such as interference with work and emotional distress. There is high comorbidity with anxiety and depression in particular (Fuller-Thomson 2008). Although there is often no clear medical explanation for established CFS, it is unclear whether this is due to failure of medical science, shortcomings in proffered medical investigations or the absence of medical underlying contributory factors. The diagnosis is difficult because there are many medical conditions that cause fatigue and many of them are obscure. Not all medical investigations are offered promptly to patients believed to have CFS (Bansal 2016). Furthermore, the nature of the fatigue is rarely, if ever, illness specific. Regarding treatment, evidence-based interventions for chronic fatigue are not curative but symptomatic and often rely on relatively small studies. Consequently, not all patients living with CFS agree with clinicians on whether strategies such as graded exercise, pacing or cognitivebehavioural therapy (CBT) actually work. Some patients quite reasonably cite potential harms and have strong individual preferences that may be at odds with their clinician's opinion. Hence there is a strong need for robust, clear and transparent studies that help patients and clinicians decide which intervention is most appropriate.

\section{About exercise therapy}

Exercise therapy is the provision of a structured course of physical activity suitable for patients with a particular disorder. Typically, this might involve a low-level aerobic exercise such as walking, jogging, swimming or cycling and/ or anaerobic exercise such as basic strength or stabilising exercises. Often it includes a negotiated, incremental increase in both duration and intensity without major decrements - socalled graded activity or graded exercise therapy (GET). From a patient's perspective it may seem odd to offer exercise therapy without addressing the cause of the fatigue. However, the cause may be elusive and there is good evidence that physical activity can help a wide variety of mental health conditions, such as depression, anxiety, bipolar disorder, schizophrenia and dementia, where the cause is similarly unknown. There is also excellent evidence that exercise improves health and quality of life for patients with many chronic diseases, such as cancer, stroke, heart failure and diabetes, where the cause may be known but irreversible. The evidence that exercise therapy does any harm is slim, but acceptability is a problem. In CFS, by definition, fatigue and/or depression directly limit involvement in and motivation for exercise therapy. But the same can be said of patients with
Alex J. Mitchell is Professor of Psycho-Oncology and Liaison Psychiatry at the University of Leicester. Correspondence Dr Alex J. Mitchell, Department of PsychoOncology, Hadley House, Leicester General Hospital, Leicester LE5 4PW UK.Email ajm80@le.ac.uk

Copyright and usage (C) The Royal College of Psychiatrists 2017.

${ }^{\dagger}$ See p. 144, this issue. 
cancer, and exercise participation rates in cancer are acceptable (Dennett 2016). The key is support, encouragement and monitoring of the programme (Castell 2011; Firth 2016). Some patients struggle with GET, but many object to the offer of CBT, particularly when it is given in a mental health setting as an alternative (rather than an adjunct) to medical care.

\section{The Cochrane review}

This Cochrane review (Larun 2016) updates an original review published in 2004 (Edmonds 2004). The 2004 review included five studies and the current version adds two modestly sized new studies (Jason 2007; Wearden 2010) and one largescale study, the PACE trial (White 2011). The three added studies were complex four-arm (Jason 2007; White 2011) and three-arm designs (Wearden 2010) with control intervention arms (respectively relaxation plus flexibility exercises, treatment as usual, and waiting list). In the PACE study, the control arm received specialist medical care alone (effectively, treatment as usual), while the treatment arms received specialist medical care plus CBT, GET or adaptive pacing therapy. Thus, Larun et al's update included eight studies, which randomly assigned a total of 1518 participants with relatively mild CFS to various study arms. Almost all of the studies reported comorbid depression, affecting between 18\% (Wearden 2010) and 39\% (Jason 2007) of participants, and this is likely to have influenced treatment response - indeed, up to $54 \%$ of patients were taking antidepressants.

Exercise therapy was typically 3-5 times per week (but for as little as $5-15$ minutes per session) over a course of 12-26 weeks. The majority of the studies focused on aerobic exercise reviewed by qualified therapists. Participants were usually asked to perform self-monitoring using the Borg Scale or an exercise diary, but no studies used wearable fitness monitors (such as Fitbit ${ }^{\circledR}$ ), which would have been genuinely useful for monitoring activity and sleep over a 24-hour cycle. A variety of mainly self-reported outcomes were measured, including fatigue levels, quality of life, pain, depression, sleep, anxiety and health service use. Few studies measured objective aerobic capacity such as maximal oxygen consumption $\left(\mathrm{VO}_{2}\right.$ max), although White et al (2011) did ask people to undertake a 6-minute walking test in order to examine real-world effects.

Initial results immediately following exercise were positive. That said, none of the included studies reported benefits on quality of life at the end of treatment and none found a difference in depression or anxiety. Regarding treatment comparisons, Jason et al (2007) (a total of 49 participants in the two arms) and White et al (2011) (361 participants) found no difference between GET and CBT, but Wearden et al (2010) (196 participants) found exercise superior to supportive listening. White et al (2011) found that GET was better than pacing on a number of outcomes.

Regarding drop-out rates, the Cochrane review reported that the pooled relative risk for drop-out was 1.63 (95\% CI 0.77-3.43), suggesting that dropout was a problem with GET, although it did not reach statistical significance. To understand this further it is necessary to refer to raw numbers in each study. For example, in the PACE study 136 of the 160 assigned to GET were adequately treated and 10 dropped out once treatment was underway, as compared with 142 of the 160 assigned to specialist medical care alone (14 dropped out of this arm).

\section{Controversy over PACE}

Unfortunately, the PACE study has since attracted huge controversy. Patient groups have long been critical of the CFS concept (as well as CFS trials in general), but the criticism of the PACE trial came from both patients and professionals. The matter could have been easily resolved if the original authors had issued a prompt correction or released suitably anonymised primary data. However, both options were repeatedly refused by the investigators and by the principal research institution, Queen Mary University of London (QMUL) (Geraghty 2016). Even when directed to release data by the Information Commission for England, QMUL spent $£ 200000$ on legal fees in a meaningless appeal (Savage 2016). Under court order, the PACE study's authors finally were required to release their raw data in September 2016 and it is now publicly available (https://sites.google.com/site/pacefoir/paceipd_foia-qmul-2014-f73.xlsx?attredirects=0). In an odd move, the day before the release, the PACE authors released a re-analysis of their own data (Goldsmith 2016). This appeared to be a late response to criticism of deviation from their original protocol method, namely, that they used bimodal and dichotomous scoring rather than continuous and/or Likert scoring. Following the release of the raw data, several patients and independent scientists collaborated to analyse the data and posted their findings online (Racaniello 2016). It is important to remember that releasing individual patient data does not correct any prior methodological flaws - it simply opens the data up for transparent re-interpretation. For example, in this case it is also alleged that the investigators 
(perhaps inadvertently) influenced participants' self-reports with indiscriminate encouragement in newsletters sent out during the trial. It is also alleged that the investigators switched their own scoring methods mid-trial.

Despite these wide criticisms, re-analysis of the PACE primary data appears to show largely the same findings (which will satisfy advocates of PACE), but at a much lower magnitude of effect (which will satisfy detractors of PACE). Even a minor change in outcome raises questions for the validity of this Cochrane review. That said, this public re-analysis might not invalidate the Cochrane meta-analysis, because Larun et al predominantly relied on statistical means and standard deviations for each symptom measure outcome. (These statistics were not revised after primary data were finally released.) It should be noted that these symptom measures are probably the least meaningful type of statistical data. They demonstrate differences between groups, but not how many individuals improved or even the percentage of improvement. In short, this Cochrane review shows that CFS symptoms improve with exercise therapy, but not how many patients recover or how many can function day to day or even how many feel better. It is useful therefore to note that objective metrics from the 6-minute walking test data were made public in the raw data released by White et al in 2016 (Fig. 1). Results show that there appears to be a very disappointing baseline 6-minute walking ability in all groups (i.e. before treatment) and a rather disappointing increase in all groups after treatment, although this is actually statistically significant (but still very modest) for those receiving GET.

Independent re-analysis examined data for recovery at the end of the trial and findings were also disappointing (Matthees 2016). The recovery rates using a priori thresholds were as follows: 3.1\% for specialist medical care alone, $6.8 \%$ for CBT, $4.4 \%$ for GET and $1.9 \%$ for adaptive pacing therapy, with no significant differences between groups. The PACE authors themselves maintained that CBT and GET were associated with significantly increased recovery rates of $22 \%$ at 52 -week followup, compared with only $8 \%$ for adaptive pacing therapy and 7\% for specialist medical care alone (White 2013). Both reports were different from the editorial claims that appeared in the $B M J$ at the time of initial publication of the PACE study, which suggested that $28-30 \%$ of patients recover using CBT and GET (Knoop 2011).

Long-term follow-up at 2.5 years found that any differences apparent between treatment arms at 52 weeks were lost as adaptive pacing and



FIG 1

Data from White et al (2011), released in full in Goldsmith et al (2016).

specialist medical care caught up with CBT and GET (Sharpe 2015).

\section{Conclusions}

At face value the overall findings are that exercise therapy is somewhat effective for CFS, particularly when compared with treatment as usual, in that it reduces symptoms at the end of therapy, possibly with some sustained benefits. The hidden detail is, as usual, rather more complicated. Exercise therapy is probably the most effective of the modalities studied in terms of daily function, as measured by a walking test, but results are so poor that, despite being statistically significant, they are no cause for celebration. Recovery rates are similarly disappointing. Independent reanalysis of the PACE data found that only about $3 \%$ recover with standard medical care, which tells us that standard medical care is not working adequately for patients with CFS and we need to re-examine why it is so ineffective. Only about $4-7 \%$ of patients recover in active treatment over 3-6 months, which is a significant improvement in terms of relative risk, but not in terms of absolute risk change.

Beyond the raw results this controversy has a number of critical lessons. First and foremost, it is imperative for researchers to publish studies in the most open and transparent manner possible. This may include responding to requests for methodological clarification, requests for reanalysis and even requests for primary data. In some online journals there is actually a requirement to release such data on request. It is remarkable that it is still not normal practice for researchers to reveal or copublish their actual anonymised raw data. A second lesson is that clinicians and researchers should work more closely with patients in both study design and 
study interpretation. Clinicians and academics may not have the same views on what is and is not acceptable therapy for patients. The third lesson is that, to promote acceptability, psychosocial treatments should be integrated into medical care. In practical terms this means that patients should be offered these options as an optional add-on while in medical care, not as a way of discharging patients perceived as difficult into a mental health service. One major reason for low parity of esteem is that physical concerns are overlooked in patients with mental health complications. May patients with CFS need psychological support and, where necessary, mental health input, but not at the expense of thorough medical care.

\section{References}

Bansal AS (2016) Investigating unexplained fatigue in general practice with a particular focus on CFS/ME. BMC Family Practice, 17: 81.

Castell BD, Kazantzis N, Moss-Morris RE (2011) Cognitive behavioral therapy and graded exercise for chronic fatigue syndrome: a metaanalysis. Clinical Psychology: Science and Practice, 18: 311-24.

Dennett AM, Peiris CL, Shields N, et al (2016) Moderate-intensity exercise reduces fatigue and improves mobility in cancer survivors: a systematic review and meta-regression. Journal of Physiotherapy, 62: 68-82.

Edmonds M, McGuire $\mathrm{H}$, Price $\mathrm{J}$ (2004) Exercise therapy for chronic fatigue syndrome. Cochrane Database of Systematic Reviews, 3: CD003200

Firth J, Rosenbaum S, Stubbs B, et al (2016) Motivating factors and barriers towards exercise in severe mental illness: a systematic review and meta-analysis. Psychological Medicine, 46: 2869-81.

Fuller-Thomson E, Nimigon J (2008) Factors associated with depression among individuals with chronic fatigue syndrome: findings from a nationally representative survey. Family Practice, 25: 414-22
Geraghty KJ (2016) 'PACE-Gate': When clinical trial evidence meets open data access. Journal of Health Psychology, Nov 1. pii: doi 10.1177/1359105316675213 [Epub ahead of print]

Goldsmith KA, White PD, Chalder T, et al (2016) The PACE Trial: Analysis of Primary Outcomes using Composite Measures of Improvement. Wolfson Institute of Preventive Medicine, Queen Mary University of London (http://www.wolfson.qmul.ac.uk/images/pdfs/pace/PACE_ published_protocol_based_analysis_final_8th_Sept_2016.pdf).

Jason L, Torres-Harding S, Friedberg F, et al (2007) Non-pharmacologic interventions for CFS: a randomized trial. Journal of Clinical Psychology in Medical Settings, 14: 275-96.

Knoop H, Bleijenberg G (2011) Chronic fatigue syndrome: where to PACE from here? Lancet, 377: 786-8.

Larun L, Brurberg KG, Odgaard-Jensen J, et al (2016) Exercise therapy for chronic fatigue syndrome. Cochrane Database of Systematic Reviews, 6 : CD003200. doi: 10.1002/14651858.CD003200.pub6

Matthees A (2016) Comment on 'Freedom of information: can researchers still promise control of participants' data?'. PubMed (https://www.ncbi. nlm.nih.gov/myncbi/alem.matthees.1/comments/2016).

Racaniello V (2016) No 'recovery' in PACE trial, new analysis finds. Virology Blog, 21 September (http://www.virology.ws/2016/09/21/norecovery-in-pace-trial-new-analysis-finds).

Savage A (2016) Judge dismisses QMUL's appeal, University must release PACE data. The Print 3 October (http://theprintnews.co.uk/2016/10/ judge-dismisses-qmuls-appeal-university-must-release-pace-data).

Sharpe MD, Goldsmith KA, Johnson AL, et al (2015) Rehabilitative treatments for chronic fatigue syndrome: long-term follow-up from the PACE trial. Lancet Psychiatry, 2: 1067-74.

Wearden AJ, Dowrick C, Chew-Graham C, et al (2010) Nurse led, home based self help treatment for patients in primary care with chronic fatigue syndrome: randomised controlled trial. BMJ, 340: c1777.

White PD, Goldsmith KA, Johnson AL, et al (2011) Comparison of adaptive pacing therapy, cognitive behaviour therapy, graded exercise therapy, and specialist medical care for chronic fatigue syndrome (PACE): a randomised trial. Lancet, 377: 823-36.

White PD, Goldsmith K, Johnson AL, et al (2013) Recovery from chronic fatigue syndrome after treatments given in the PACE trial. Psychological Medicine, 43: 2227-35. 